\title{
Inelastic Production of $J / \psi$ Mesons in Photoproduction and Deep Inelastic Scattering at HERA
}

\author{
Michael Steder* ${ }^{\dagger}$ \\ DESY, Hamburg \\ E-mail: michael.steder@desy.de
}

\begin{abstract}
Inelastic photo- and electroproduction of $J / \psi$ mesons is studied in $e p$ scattering at HERA. The data were recorded with the $\mathrm{H} 1$ detector in the period from 2004 to 2007. Single and double differential cross sections are determined and helicity distributions of the $J / \psi$ mesons are analysed. The results are compared to theoretical predictions in the colour singlet model and in the framework of non-relativistic QCD. Calculations in the colour singlet model using a $k_{T}$ factorisation ansatz describe the data well, while colour singlet model calculations to next-to-leading order in collinear factorisation underestimate the data.
\end{abstract}

XVIII International Workshop on Deep-Inelastic Scattering and Related Subjects April 19 -23, 2010

Convitto della Calza, Firenze, Italy

*Speaker.

${ }^{\dagger}$ on behalf of the $\mathrm{H} 1$ collaboration 


\section{Introduction}

Charmonium production in electron ${ }^{1}$ proton collisions at HERA is dominated by photon-gluon fusion: a photon emitted from the incoming electron interacts with a gluon from the proton to produce a $c \bar{c}$ pair that evolves into a charmonium state. In the colour singlet model (CSM) [1], only those states with the same quantum numbers as the resulting charmonium contribute to the formation of a bound $c \bar{c}$ state. This is achieved by radiating a hard gluon in a perturbative process. In the factorisation ansatz of non-relativistic quantum chromodynamics (NRQCD) [2], also colour octet $c \bar{c}$ states contribute to the charmonium production cross section via soft gluon radiation. At HERA two kinematic domains are distinguished: In photoproduction $\left(Q^{2} \lesssim 2 \mathrm{GeV}^{2}\right)$ the photons are quasi-real, in electroproduction they have a higher virtuality $\left(Q^{2}>3.6 \mathrm{GeV}^{2}\right)$.

This paper summarises the results of a measurement of inelastic $J / \psi$ meson production with the $\mathrm{H} 1$ detector at HERA published in [3]. The $J / \psi$ meson candidates are identified by their decay into two leptons.

\section{Cross Section Measurement}

The cross section measurement is performed in a kinematic range in which contributions from diffractive charmonium production are suppressed, $60<W_{\gamma p}<240 \mathrm{GeV}, P_{T, \psi}\left(P_{T, \psi}^{*}\right)>1 \mathrm{GeV}$ and $0.3<z<0.9$, where $z=\left(p_{\psi} \cdot p\right) /(q \cdot p)$ denotes the elasticity of the $J / \psi$ mesons. The photon virtuality $Q^{2}$ is limited in the electroproduction analysis to $3.6<Q^{2}<100 \mathrm{GeV}^{2}$ and for the photoproduction sample to $Q^{2}<2.5 \mathrm{GeV}^{2}$. The measured cross sections are not corrected for QED radiative effects and for contributions originating from diffractive $\psi(2 S)$ mesons and $b$ hadrons. Bin-centre corrections using MC simulations are applied.

For the photoproduction sample the measured $e p$ cross sections are transformed to $\gamma p$ cross sections using photon flux factors, calculated in the Weizsäcker-Williams approximation [4]. Differential $J / \psi$ meson photoproduction cross sections are presented in figure 1(a,b) as function of the $z$ and the $P_{T, \psi}^{2}$ of the $J / \psi$ meson, showing a reasonable agreement with the prediction from the CASCADE MC generator [5]. A variation of the renormalisation scale in the MC simulation by a factor of two $\left(0.5 \mu_{0}<\mu_{\mathrm{r}}<2 \mu_{0}\right)$ has little effect as shown by the band in the figures. In addition to the CASCADE prediction, the remaining contributions from diffractive $\psi(2 S)$ mesons and from $b$ hadrons are shown. At low $z$ and low transverse momenta of the $J / \psi$ mesons, CASCADE overshoots the data, while CASCADE is below the data at large $z$ and large transverse momenta. Taking into account that the measured cross section in the lowest $z$ bin includes about $20 \%$ of events originating from $b$ hadron decays, the difference to the CASCADE prediction is even larger. For electroproduction, the differential $e p$ cross section as functions of the photon virtuality $Q^{2}$ is shown in figure 1(c). A comparison with predictions from CASCADE reveals an overall reasonable agreement with the data.

For photoproduction, several NLO calculations have been performed and are compared with the data in figure 2. A calculation in the CSM at NLO [6] was repeated using up-to-date sets of scale parameters [7, 8], yielding predictions as shown in figure 2(a)-(b). The shapes of the data are quite well described, whereas the normalisation of the prediction is about a factor three below the data,

\footnotetext{
${ }^{1}$ Here "electron" is used to denote both electron and positron.
} 
(a)

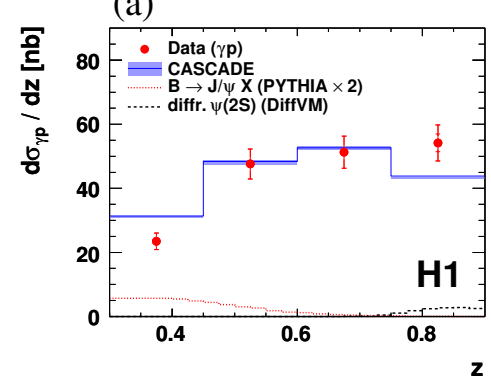

(b)

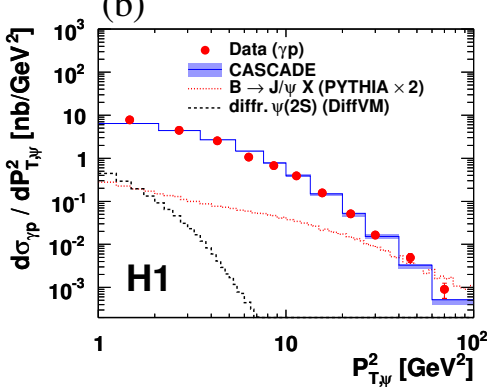

(c)

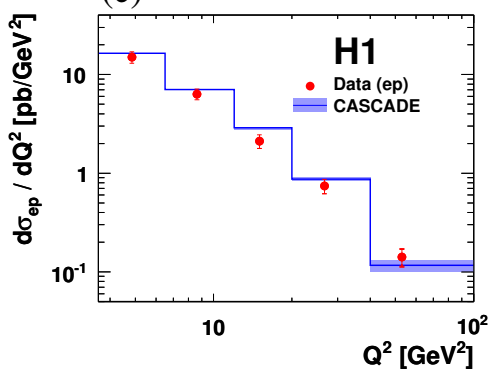

Figure 1: (a,b) Differential $J / \psi$ meson photoproduction $\left(Q^{2} \lesssim 2 \mathrm{GeV}^{2}\right)$ and (c,d) electroproduction $(3.6<$ $Q^{2}<100 \mathrm{GeV}^{2}$ ) cross sections as functions of (a) $z$, (b) $P_{T, \psi}^{2}$, (c) $Q^{2}$ and (d) $z$. The data are compared to the predictions from CASCADE (solid line). The dashed and dotted lines indicate the remaining background from diffractive $\psi(2 S)$ or $b$ hadron decays respectively as estimated using MC simulations.

with large uncertainties, indicating that corrections beyond next-to-leading order are necessary in order to describe the data.

The calculation to next-to-leading order has been extended to include colour octet contributions resulting in a larger cross section [8]. The data are compared to this prediction in figure 2(c)-(d). The dominant theory uncertainty arises from the difference in the predicted cross section when using LO colour octet long distance matrix elements (LDMEs) or higher order improved LDMEs [8]. The NRQCD prediction fails however in describing the shape of the differential cross section as a function of the $z$, even within the presently large uncertainties of the calculation. In addition to these uncertainties, the reliability of NRQCD calculations is questionable at large values of $z$, due to the presence of both large perturbative corrections and enhanced non-perturbative power corrections [11].

\section{Polarisation Measurement}

The measurement of the $J / \psi$ meson helicity distributions provides an independent method to distinguish between different production mechanisms. The measurement is performed for the photoproduction data sample. The $J / \psi$ meson polarisation is measured by analysing the decay angle distributions of the $J / \psi$ meson in two complementary frames: the helicity frame and the Collins-Soper frame. The frame-dependent polarisation axis is taken as $z$ axis of a right handed coordinate system, where the $x$ and $z$ axis lie in a plane spanned by the photon and proton directions. The $y$ axis is perpendicular to this plane and is the same in both reference frames. The polar $\left(\Theta^{*}\right)$ and azimuthal $\left(\phi^{*}\right)$ angles of the positive decay muons are used to parametrise the measured decay angle distributions as function of $\cos \left(\Theta^{*}\right)$ and $\phi^{*}$ [12]: $\mathrm{d} \sigma / \mathrm{d} \cos \Theta^{*} \propto 1+\alpha \cos ^{2} \Theta^{*}$ and $\mathrm{d} \sigma / \mathrm{d} \phi^{*} \propto 1+\frac{\alpha}{3}+\frac{v}{3} \cos 2 \phi^{*}$. The polarisation variables $\alpha$ and $v$ can be related to elements of the spin density matrix for the $J / \psi$ meson. Moreover, $\alpha=+1$ and -1 corresponds to fully transverse and longitudinal polarisation of the $J / \psi$ meson, respectively. A $\chi^{2}$ fit is performed in each bin of the polarisation measurement, comparing data to Monte Carlo samples on reconstruction level probing values for $\alpha$ and $v$ between -1 and +1 . Systematic uncertainties on this measurement are negligible compared to rather large statistical uncertainties. The results for $\alpha$ as a function of $P_{T, \psi}$ and $v$ as a function of $z$ are presented for both frames in figure 3 . 
(a)
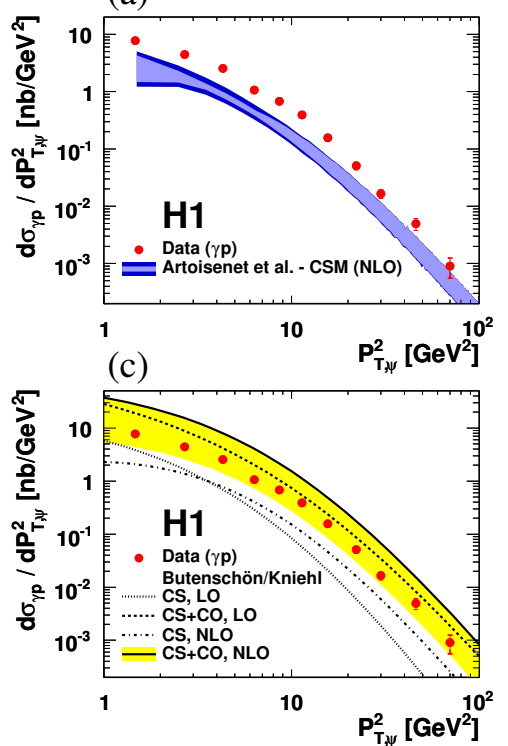

(b)

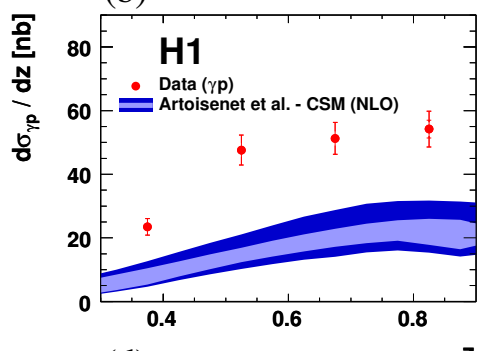

(d)

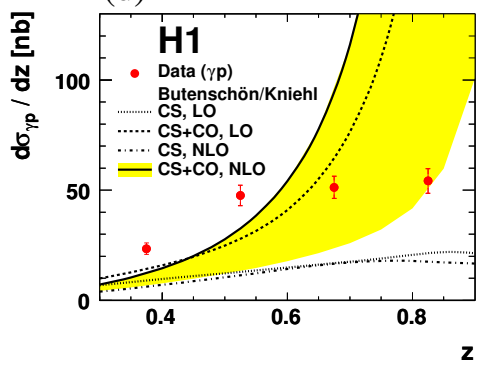

Figure 2: Differential $J / \psi$ meson photoproduction cross sections as functions of $P_{T, \psi}^{2}$ (a) and c)) and $z$ (b) and d)). The data are compared with calculations to next-to-leading order: a,b) a colour singlet model (CSM) calculation [7] and c,d) a NRQCD calculation including contributions from colour octet states (CS + $\mathrm{CO}$ ) [8]. The colour singlet component (CS) of the latter calculation is shown separately in addition.

Within experimental uncertainties, $J / \psi$ mesons produced inelastically at HERA are unpolarised. The measurements are compared to predictions using a $k_{T}$ factorisation ansatz [13] and to calculations in the CSM in collinear factorisation at leading order [13] and next-to-leading order [7]. The predictions in the $k_{T}$ factorisation ansatz describe the data. The NLO calculations show a similar trend within large uncertainties. In contrast, the leading order CSM calculation predicts larger values for the polarisation variables and is disfavoured by the measurement.

\section{Conclusions}

A measurement by the $\mathrm{H} 1$ collaboration [3] of inelastic $J / \psi$ meson production in ep scattering is summarised. The measured differential cross sections and the obtained polarisation parameters are compared to a number of recent theory predictions. It is found that predictions based on $k_{T}$ factorisation in the colour singlet model are able to describe the cross sections and the helicity distributions well. Calculations based on collinear factorisation in the colour singlet model at nextto-leading order produce a reasonable description of the shape of the measured cross sections, but are lower in normalisation. The polarisation parameter measurements are described within the large uncertainties. The failure to describe the cross section measurements and the strong sensitivity to scale variations indicate, that calculations beyond next-to-leading order are necessary. Moreover contributions from colour octet states may be significant. 

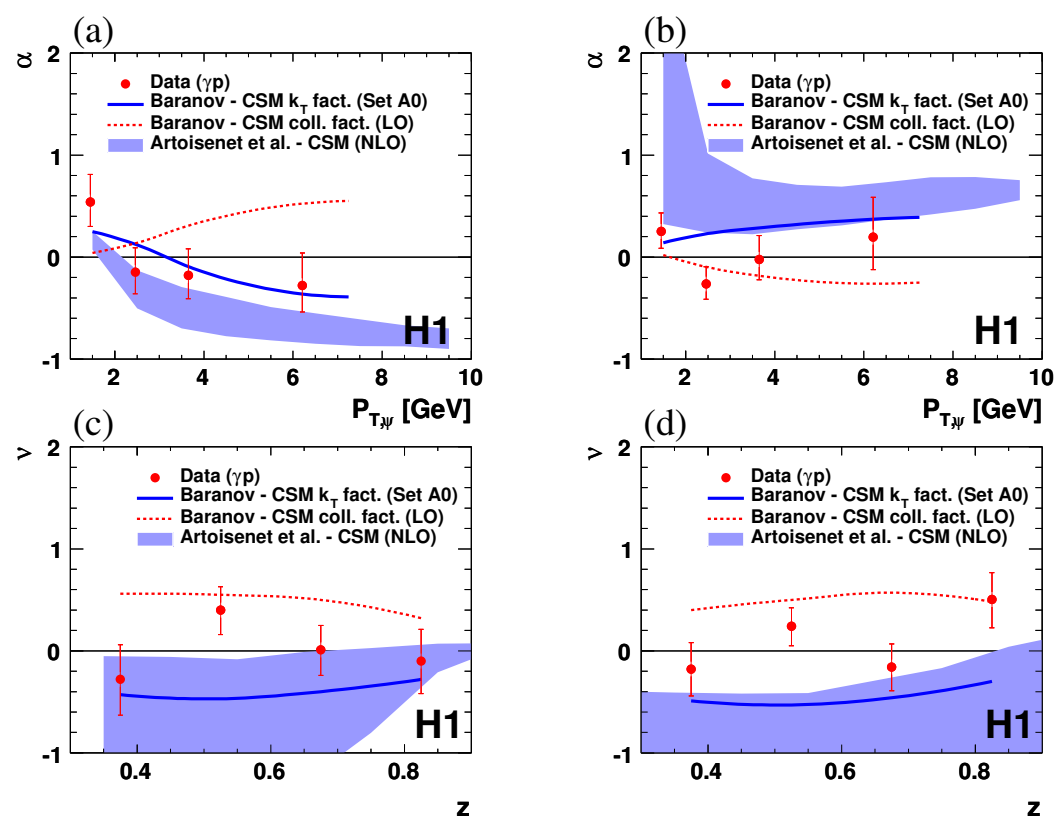

Figure 3: Polarisation parameters $\alpha(\mathrm{a}, \mathrm{b})$ and $v(\mathrm{c}, \mathrm{d})$ measured in the helicity frame $(\mathrm{a}, \mathrm{c})$ and the CollinsSoper frame (b,d) as a function of $z$ and $P_{T, \psi}$. The measurement is compared with predictions calculated in a $k_{T}$ factorisation ansatz [13] and with calculations in CSM (collinear factorisation) at LO [13] and NLO [7].

\section{References}

[1] C. H. Chang, Nucl. Phys. B 172 (1980) 425; E. L. Berger and D. L. Jones, Phys. Rev. D 23 (1981) 1521; R. Baier and R. Rückl, Nucl. Phys. B 201 (1982) 1; R. Baier and R. Rückl, Phys. Lett. B 102 (1981) 364; R. Baier and R. Rückl, Z. Phys. C 19 (1983) 251.

[2] W. E. Caswell and G. P. Lepage, Phys. Lett. B 167 (1986) 437; B. A. Thacker and G. P. Lepage, Phys. Rev. D 43 (1991) 196; G. T. Bodwin, E. Braaten and G. P. Lepage, Phys. Rev. D 51 (1995) 1125 [Erratum-ibid. D 55 (1997) 5853] [hep-ph/9407339].

[3] F. D. Aaron et al. [H1 Collaboration], Eur. Phys. J. C 68 (3-4) (2010) 401 [hep-ex/1002.0234].

[4] V. M. Budnev et al., Phys. Rept. 15 (1974) 181.

[5] H. Jung and G.P. Salam, Eur. Phys. J. C 19 (2001) 351 [hep-ph/0012143]; H. Jung, Comput. Phys. Commun. 143 (2002) 100 [hep-ph/0109102].

[6] M. Krämer, Nucl. Phys. B 459 (1996) 3 [hep-ph/9508409].

[7] P. Artoisenet et al., Phys. Rev. Lett. 102 (2009) 142001 [arXiv:0901.4352].

[8] M. Butenschön and B. A. Kniehl, arXiv:0909.2798.

[9] J. M. Campbell, F. Maltoni and F. Tramontano, Phys. Rev. Lett. 98 (2007) 252002 [hep-ph/0703113].

[10] P. Artoisenet, J. P. Lansberg and F. Maltoni, Phys. Lett. B 653 (2007) 60 [hep-ph/0703129].

[11] M. Beneke, I. Z. Rothstein and M. B. Wise, Phys. Lett. B 408 (1997) 373 [hep-ph/9705286]; M. Beneke, G. A. Schuler and S. Wolf, Phys. Rev. D 62 (2000) 034004 [hep-ph/0001062]; S. Fleming, A. K. Leibovich and T. Mehen, Phys. Rev. D 74 (2006) 114004 [hep-ph/0607121].

[12] M. Beneke, M. Krämer and M. Vanttinen, Phys. Rev. D 57 (1998) 4258 [hep-ph/9709376].

[13] S. P. Baranov, private communication, 2009, based on: JETP Lett. 88 (2008) 471. 